\title{
Jogos Digitais e Engajamento na Reabilitação de Pacientes: Uma Revisão Sistemática da Literatura
}

\author{
Carlos Henrique Rorato Souza ${ }^{1}$, Daniel Machado de Oliveira ${ }^{1}$ \\ Luciana de Oliveira Berretta ${ }^{1}$, Sérgio Teixeira de Carvalho ${ }^{1}$ \\ ${ }^{1}$ Instituto de Informática - Universidade Federal de Goiás (UFG) \\ Caixa Postal 131 - 74.001-970 - Goiânia - GO - Brazil \\ ${ }^{1}$ \{carlossouza, danielmachado, luciana, sergio\}@inf.ufg.br
}

\begin{abstract}
One of the essential issues for the success of rehabilitation or telerehabilitation sessions is the patient's engagement in the activity performed. Approaches that use games to assist in the process are considered good alternatives. In this sense, this work discusses about the realization and results of a Systematic Literature Review in order to explore, identify and analyze the existing digital game projects that aim to engage the patient in the realization of the sessions. The results reaffirm problems of patient engagement and motivation (68.81\% of the works bring this problem), in addition to showing a great diversity of projects carried out, with a wide range of methods of interaction between the patient and the game, in addition to of several resources used to promote patient engagement, such as the use of the games themselves (43\%) or issues related to the difficulty of the activity (37\%).
\end{abstract}

Resumo. Uma das questões essenciais para o sucesso das sessões de reabilitação ou telerreabilitação é o engajamento do paciente na atividade realizada. Abordagens que utilizam jogos para auxiliar no processo são consideradas boas alternativas. Neste sentido, este trabalho aborda a realização e os resultados de uma Revisão Sistemática da Literatura no intuito de explorar, identificar e analisar os projetos de jogos digitais existentes que propõem-se a engajar o paciente na realização das sessões. Os resultados reafirmam problemas de engajamento e motivação dos pacientes $(68,81 \%$ dos trabalhos trazem esta problemática), além de mostrarem uma grande diversidade de projetos realizados, com uma vasta gama de métodos de interação entre o paciente e o jogo, além de diversos recursos empregados para a promoção do engajamento no paciente, como a própria utilização dos jogos em si (43\%) ou ainda questões relacionadas à dificuldade da atividade (37\%).

\section{Introdução}

O processo de reabilitação de pacientes envolve diversas questões diretamente relacionadas com a sua eficácia [Subtil et al. 2011]. Uma delas, considerada como crucial para o sucesso das sessões, é o engajamento do paciente na realização dos exercícios propostos [Jack et al. 2010]. Este pode ser entendido como um envolvimento profundo do jogador na realidade do jogo, que o deixa "imerso" nesta realidade ("estado de flow"), fazendo com que ele queira continuar dentro deste universo, sendo continuamente motivado e estimulado a assim permanecer [Csikszentmihalyi 2020, Kapp 2012, Huizinga 2020, Chou et al. 2014]. Normalmente, as atividades e exercícios de reabilitação são repetitivos, fato que desestimula o paciente, que, ou não realiza os exercícios ou os faz de maneira inadequada. Sem o engajamento e a motivação, a reabilitação não alcança os resultados esperados. 
A partir disso, muitas abordagens surgiram no intuito de despertar o engajamento no processo de reabilitação. As abordagens utilizando jogos estão entre elas, buscando tornar as atividades repetitivas menos enfadonhas e mais lúdicas, por meio da promoção de engajamento a partir dos elementos que fazem parte da natureza dos jogos [Silva et al. 2017, Tolomei 2017]. A seleção e o uso destes elementos e mecânicas, bem como os modos de interação entre o jogador e o jogo são cruciais para que se suscite a motivação e o engajamento que são esperados [Kapp 2012, Brigham 2015].

Nesse sentido, este trabalho tem por objetivo documentar uma pesquisa bibliográfica do tipo Revisão Sistemática da Literatura, realizada visando explorar, identificar e analisar os projetos de jogos digitais existentes que propõem-se a engajar o paciente na realização de sessões de reabilitação e/ou telerreabilitação, isto é, quando a atividade é realizada de forma remota, através de meios de comunicação [Santos and Pinheiro 2016, do Nascimento Piropo and do Amaral 2015]. A telerreabilitação tem se tornado cada vez mais presente no cenário de cuidados com a saúde, dadas as diversas dificuldades de disponibilidade do tratamento na forma presencial, seja por questões de acesso, de custo, ou seja ainda pelo contexto de distanciamento social imposto pela pandemia da COVID-19 [Ferguson et al. 2020, Souza et al. 2020].

Este artigo está organizado em outras quatro seções, além desta introdução. A Seção 2 apresenta a metodologia utilizada, envolvendo a definição das questões de pesquisa, palavras-chave e termos de busca, além dos critérios para a seleção dos artigos. A Seção 3, por sua vez, apresenta o processo de condução das buscas, seleção dos artigos e extração dos dados. A partir disso, a Seção 4 traz uma análise dos dados extraídos, visando trazer respostas às perguntas formuladas no início. Por fim, a Seção 5 apresenta os apontamentos finais e indica horizontes a partir das constatações feitas ao longo do processo.

\section{Metodologia da Pesquisa}

Para a condução desta pesquisa, realizou-se uma Revisão Sistemática da Literatura (RSL), seguindo a abordagem proposta por [Nakagawa et al. 2017], a qual apresenta três etapas: (I) Definição do protocolo da revisão, constituída pelas questões de pesquisa, palavraschave, sinônimos, estratégia de busca, e os critérios de inclusão e exclusão dos estudos; (II) Condução da revisão, composta pela realização das buscas, seleção dos trabalhos, extração e análise dos dados dos trabalhos selecionados; e, por fim, (III) Resultados e Discussão, que apresenta, além dos resultados, as respostas às questões de pesquisa. Para auxiliar no gerenciamento do processo, optou-se por utilizar a plataforma Parsifal ${ }^{1}$.

Esta seção apresenta, a seguir, o protocolo da revisão (questões de pesquisa, termos envolvidos na construção da string de busca e critérios de seleção). As demais etapas são descritas nas seções 3 e 4 , respectivamente.

\subsection{Questões de Pesquisa}

Tendo em vista o objetivo desta pesquisa, três questões de pesquisa $\left(Q_{1}, Q_{2}, Q_{3}\right)$ foram elaboradas:

$Q_{1}$ : "Quais são os projetos existentes que apresentam a construção de um jogo digital visando engajar o paciente na realização de sessões de reabilitação elou telerreabilitação?”. É importante conhecer as iniciativas e ferramentas que estão sendo

\footnotetext{
${ }^{1}$ https://parsif.al/
} 
desenvolvidas, pois a resposta de $Q_{1}$ tem o potencial de mostrar caminhos que têm sido explorados por pesquisadores, além de nortear possíveis horizontes de trabalho.

$Q_{2}$ : “Quais elementos de jogos são empregados para suscitar engajamento no paciente?". Dada a importância de promover o engajamento do paciente na atividade realizada, é imprescindível investigar se os projetos encontrados expressam preocupação com esta nuance, e quais elementos de jogos são utilizados neste sentido. Isso permite visualizar quais estratégias são, de fato, eficazes e qual a relevância da problemática do engajamento.

$Q_{3}$ : “Como se dá a interação entre o paciente e o jogo desenvolvido?”. É fundamental entender a forma encontrada pelos projetos para estabelecer a interação entre o paciente e o jogo. Formas invasivas ou incômodas podem desestimular o paciente, diminuindo a adesão ao tratamento ou tornando a atividade desconfortável, enquanto abordagens que utilizam sensores e equipamentos de fácil uso, aprendizado e configuração podem ser melhor aceitas [Bassett 2003].

\subsection{Palavras-Chave, Sinônimos e Estratégia de Busca}

As palavras a seguir foram selecionadas para a pesquisa, dada a sua alta incidência na literatura científica dedicada à área: (P.1) engagement (adherence, engaging); (P.2) game (exergame, exergaming, games); e (P.3) rehabilitation (telerehabilitation, tele rehabilitation, tele-rehabilitation).

Assim, a string de busca genérica utilizada para as buscas é: ("game" OR "exergame" OR "exergaming" OR "games") AND ("rehabilitation" OR "telerehabilitation" $O R$ "tele rehabilitation" OR "tele-rehabilitation") AND ("engagement" OR "adherence" OR "engaging”), considerando disjunções entre os sinônimos e conjunções entre as palavraschave.

Em relação à estratégia para a realização da revisão, optou-se por uma busca diretamente nas bases bibliográficas selecionadas: ACM Digital Library ${ }^{2}$, Scopus $^{3}$ e IEEE Digital Library $^{4}$, por serem os principais acervos que se relacionam com a área de Computação e com o desenvolvimento de jogos digitais, e PubMed ${ }^{5}$, por relacionar-se à área da saúde.

\subsection{Critérios de Inclusão e Exclusão}

De acordo com [Nakagawa et al. 2017, Sampaio and Mancini 2007], para verificar a relevância dos estudos encontrados, a aplicação de critérios de inclusão e exclusão é processo indispensável em uma RSL. Nesta pesquisa, foram adotados como critérios de inclusão duas características inerentes à problemática em questão, enumeradas a seguir. Se a resposta a alguma das duas perguntas foi positiva, o trabalho foi incluído.

1. O trabalho apresenta a construção de um jogo para a reabilitação?

2. O trabalho apresenta projeto sobre jogos em telerreabilitação?

Por outro lado, para a exclusão dos estudos foram adotados diversos critérios, que foram sendo refinados ao longo do processo de condução. Foi excluído o trabalho que se encaixou em algum dos seguintes critérios:

1. Não apresenta nenhuma das palavras-chave no título e/ou resumo;

\footnotetext{
${ }^{2}$ http://portal.acm.org

${ }^{3} \mathrm{http}: / /$ www.scopus.com

${ }^{4} \mathrm{http}$ ://ieeexplore.ieee.org

${ }^{5}$ https://pubmed.ncbi.nlm.nih.gov/
} 
2. Não apresenta o desenvolvimento de um jogo digital;

3. Não apresenta avaliação e resultados;

4. Não é artigo científico;

5. Não está escrito nas línguas portuguesa ou inglesa;

6. Não está inserido no contexto de fisioterapia/reabilitação;

7. Não possui resumo e título;

8. Texto integral do trabalho não acessível; e

9. Trabalho possui data de publicação anterior a 5 anos.

Assim foi possível determinar, dentre os trabalhos encontrados a partir da string de busca desenvolvida, quais atendem aos propósitos desta pesquisa e quais são considerados relevantes para responder às questões apresentadas.

\section{Condução da Revisão}

Com o planejamento estabelecido, teve início a etapa de Condução, que compreendeu a realização das buscas, a seleção dos estudos, extração e ainda a análise dos dados extraídos.

O primeiro passo, portanto, foi a realização das buscas, em que a string de busca foi aplicada às bases selecionadas para a obtenção dos resultados, os quais foram catalogados na plataforma Parsifal. Durante o processo, a string foi adaptada (em termos de sintaxe) a cada base, levando-se em conta as particularidades exigidas por cada uma.

A seguir se deu a seleção dos estudos. Neste momento, as referências bibliográficas encontradas nas bases foram avaliadas, tendo em vista os critérios de inclusão e exclusão utilizados para classificar os trabalhos em aceito ou rejeitado. De maneira geral, isso se deu a partir das leituras do título, palavras-chave e resumo dos artigos. Em alguns casos, entretanto, o texto integral precisou ser consultado.

O resultado desta seleção está disposto na Tabela $1^{6}$. Nela, é possível visualizar a quantidade de artigos encontrados nas buscas em cada base, além da quantidade de trabalhos aceitos e rejeitados. No total, 93 estudos foram selecionados, considerando o descarte de trabalhos duplicados. Todos estes artigos foram selecionados para a leitura completa, visando a extração e análise dos dados. O critério de exclusão que foi aplicado de forma mais frequente para a rejeição dos trabalhos foi o de número 2, "não apresenta o desenvolvimento de um jogo digital".

Tabela 1. Artigos aceitos e rejeitados por base de dados.

\begin{tabular}{lccc}
\hline & Encontrados & Aceitos & Rejeitados \\
\hline ACM & 389 & 29 & 360 \\
\hline IEEE & 164 & 38 & 126 \\
\hline Scopus & 118 & 11 & 107 \\
\hline PubMed & 249 & 15 & 234 \\
\hline
\end{tabular}

Finalizada a seleção, foi realizada a leitura completa e a extração dos dados dos artigos selecionados. Este processo pode ocorrer de diversas formas, podendo ser, por

\footnotetext{
${ }^{6}$ Um apêndice contendo a Tabela completa e todas as referências bibliográficas dos trabalhos selecionados foi, por questões de espaço, disponibilizado para o acesso em https://bit.ly/3yz0jqZ.
} 
exemplo, a partir da elaboração de fichas de leitura ou resumos/sínteses críticas com informações que são consideradas pertinentes para a resposta às questões de pesquisa [Nakagawa et al. 2017, Sampaio and Mancini 2007]. A plataforma Parsifal apresenta a possibilidade de criação dessas fichas de leitura por meio de formulários, cujas questões são elaboradas pela equipe que conduz a revisão. Nesta RSL, optou-se por utilizar este recurso. O questionário elaborado está disposto a seguir.

- Qual a abordagem proposta pelo artigo?

- Como o projeto pretende criar engajamento no paciente?

- Qual o problema/disfunção/doença abordado no trabalho?

- Quais as dificuldades existentes que justificam a construção do jogo?

- Como acontece a interação entre paciente, fisioterapeuta e o jogo?

- Qual o protocolo utilizado para a testagem/validação do projeto?

- De 0 (pouco eficiente/eficaz) a 10 (muito eficiente/eficaz), qual o índice de eficácia da solução proposta pelo trabalho, com base nos resultados dos testes?

- Envolve testes com pacientes?

- Contou com o acompanhamento de profissionais de saúde durante a construção da solução apresentada?

As respostas do questionário permitiram, após etapas de análise e um processo de interpretação, a elaboração de respostas às perguntas de pesquisa.

\section{Resultados e Discussão}

Os 93 artigos encontrados apresentam diversas abordagens envolvendo jogos digitais, reabilitação de pacientes e a geração de engajamento. Uma análise do perfil dos trabalhos é o primeiro passo, dada a quantidade de artigos, visando identificar tendências e alguns padrões entre os trabalhos.

Sobre o ano de publicação, o gráfico disposto na Figura 1 mostra a distribuição dos trabalhos aceitos pelos anos considerados para a seleção (os cinco últimos anos). Nota-se um crescimento considerável a partir do ano de 2017. O ano de 2019 apresenta o maior número de publicações, somando, no total, 26 artigos selecionados.

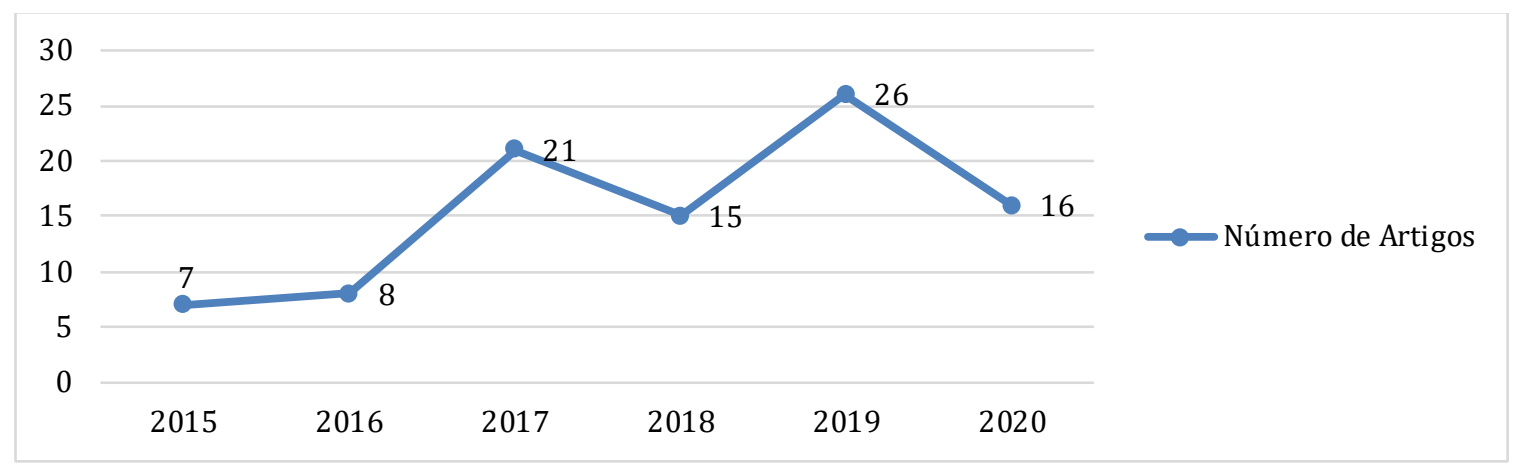

Figura 1. Artigos aceitos separados por ano de publicação.

\section{1. $\left(Q_{1}\right)$ Quais são os projetos existentes que apresentam a construção de um jogo digital visando engajar o paciente na realização de sessões de reabilitação e/ou telerreabilitação?}

Há uma grande diversidade de projetos. Para uma resposta completa a esta pergunta, seria necessária uma discussão sobre cada trabalho encontrado, suas peculiaridades e singularidades. Algumas características são discutidas nas respostas às outras questões $\left(Q_{2}\right.$ e $\left.Q_{3}\right)$. É possível, entretanto, identificar linhas comuns, que podem ser apontamentos a esta pergunta. 
Um primeiro ponto é o problema de saúde abordado: tratam-se de disfunções motoras, resultado de grandes períodos de internação, disfunções musculares por Acidentes Vasculares Cerebrais (AVCs), ou ainda pela idade avançada do paciente. Uma grande porção dos artigos dedica-se aos movimentos dos membros superiores $(50,5 \%)$ [Lai et al. 2018, Pereira et al. 2019, Bower et al. 2015], enquanto que 9,6\% aos membros inferiores e ainda 39,7\% dos trabalhos, a ambos ou outros tipos de movimentação [Grammatikopoulou et al. 2019, Desai et al. 2016]. Por essa razão, é unanimidade entre os trabalhos encontrados que o jogo construído é um exergame, ainda que alguns não sejam formalmente conceituados desta maneira. Exergames são jogos sérios que visam motivar a realização de atividades físicas [Oh and Yang 2010].

Outro ponto importante é a justificativa que suscitou nos autores a criação dos jogos. A falta de motivação dos pacientes (os exercícios são repetitivos e tediosos) aparece como primeiro fator em 68,8\% dos artigos encontrados [Chebaa et al. 2016, Eisapour et al. 2020, Dhawan et al. 2019]. Outros fatores ainda considerados são o alto custo dos tratamentos disponíveis $(15 \%)$ ou ainda outros fatores $(26,8 \%$ ), como as dificuldades de acesso [Afyouni et al. 2019, Cuthbert et al. 2019].

Já em relação ao uso de abordagens envolvendo telerreabilitação, tem-se que poucos trabalhos foram encontrados que tragam esta questão de forma direta (cerca de $12 \%$ ou 11 trabalhos) [Tamayo-Serrano et al. 2020, David et al. 2018]. Entretanto, notase uma grande precoupação no contexto de reabilitação em casa ("home based" rehabilitation), o que é um passo importante na direção da telerreabilitação [Corrêa et al. 2019, Vasconcelos et al. 2018], ainda que estas abordagens considerem apenas o cenário pacientejogo, desconsiderando intervenções do profissional de saúde. A maioria dos projetos, todavia, necessita da presença física do terapeuta, que orienta o uso dos aparelhos e realiza a avaliação do paciente [Bortone et al. 2018, Ferreira and Menezes 2020, Funabashi et al. 2017].

A participação direta de profissionais de saúde no processo é outro ponto que pode trazer respostas. Cerca de $84,9 \%$ dos trabalhos contaram com o acompanhamento de profissionais de saúde durante o processo de construção ou validação da solução [Agres and Herremans 2017, Burdea et al. 2020]. Ao serem observadas, entretanto, as etapas de desenvolvimento dos trabalhos e os protocolos utilizados nos testes, percebe-se que o envolvimento destes profissionais ainda é limitado na maioria das iniciativas às etapas finais (de validação) dos projetos, não sendo incluídos desde o início.

Por fim, questões relacionadas aos testes e avaliação dos jogos podem ser citadas. Um total de $69,8 \%$ dos trabalhos apresenta testagens com pacientes, que aconteceram sob os mais diversos protocolos e etapas [Huerga et al. 2016, David et al. 2017]. No geral $(91,3 \%)$ foram realizados testes de usabilidade, onde o grupo de pacientes é levado a testar a aplicação durante um determinado tempo e depois responde questionários sobre a experiência de uso [Pedroli et al. 2017, Barioni et al. 2017, Tamayo-Serrano et al. 2020].

Pode-se afirmar que os projetos que envolvem jogos lidam, na sua maior parte, com disfunções motoras por meio de exergames desenvolvidos e testados com pacientes. A criação destes jogos é também resposta a dificuldades de custo, acesso e, principalmente de engajamento do paciente nas atividades realizadas. Entretanto, a participação direta dos profissionais de saúde ainda é limitada durante o processo, dado que, quando são envolvidos, o são apenas nas etapas finais de validação. 


\section{2. $\left(Q_{2}\right)$ Quais elementos de jogos são empregados para suscitar engajamento no paciente?}

Para os apontamentos sobre a questão $Q_{2}$, foi construído o gráfico disposto na Figura 2 (a). A geração de engajamento no paciente é tema relevante nos trabalhos encontrados, dado que a sua falta é atestada como um grande problema e é ainda constatada como sendo um fator que, por si só, motiva a construção de muitos dos projetos.

Os trabalhos apresentam diversas soluções para vencer este obstáculo. Grande parte dos projetos (51 trabalhos ou 43\%) afirma que a própria construção do jogo já se coloca como um fator promotor de engajamento [Rahman et al. 2016, Chatzitofis et al. 2015, Madrigal et al. 2019, Bower et al. 2015]. Estes trabalhos não citam elementos específicos colocados nos jogos para que esse engajamento seja efetivo.

Alguns outros trabalhos, porém, detalham o uso de técnicas e recursos específicos. 19 artigos (37\%) defendem que a dificuldade da tarefa a ser realizada no jogo é fator que prende a atenção do usuário [Ferreira et al. 2019, Chen et al. 2020, Ferreira and Menezes 2020]. A atividade não pode ser exageradamente difícil ou extremamente fácil para o jogador, pois ele perde o interesse e desiste de permanecer jogando. $\mathrm{O}$ ideal, segundo estas abordagens, é permitir ou realizar a regulagem da dificuldade, quer seja antes da realização da atividade, quer durante - de forma manual ou automática.

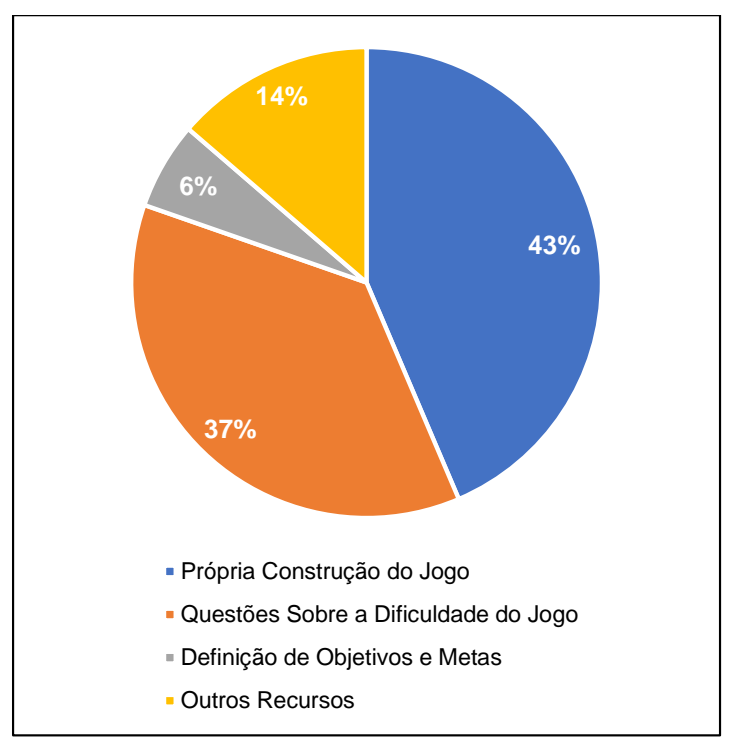

(a) Recursos para a geração de engajamento.

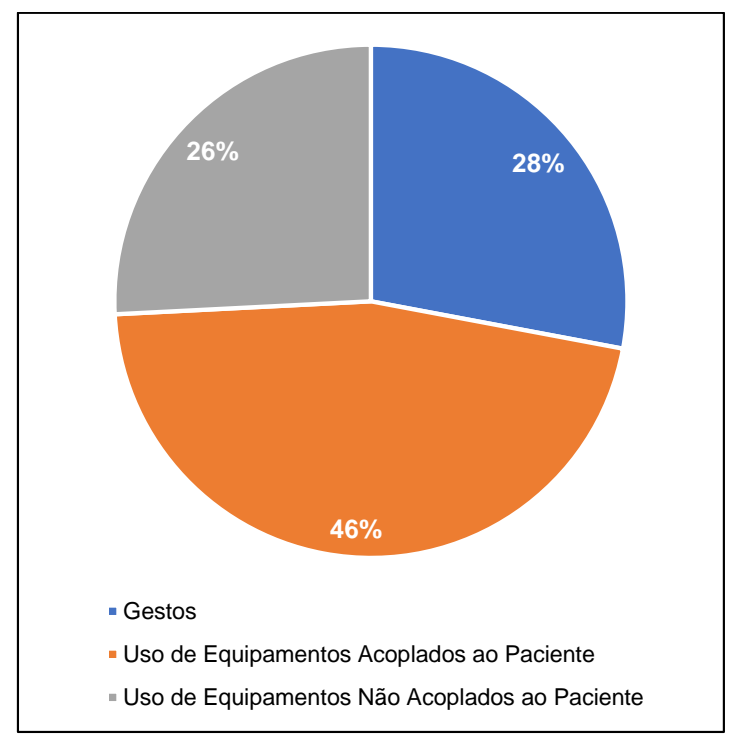

(b) Abordagens de interação.

Figura 2. Dados acerca dos resultados encontrados na revisão.

De todos os artigos encontrados, 7 deles (6\%) propõem que a definição de objetivos e metas é um importante recurso para o engajamento [Rodrigues et al. 2019, Uzor and Baillie 2019]. Colocar "alvos" a serem alcançados a curto e médio prazo, bem como premiações e elementos bônus, é algo que faz com que o jogador queira continuar envolvido na atividade, ganhando e conquistando cada vez mais. Os sistemas de pontuação e ranqueamento entram neste conceito. O senso de competitividade, consigo mesmo ou com outros, é ativado neste caso, e é fator que estimula a vontade de realizar a reabilitação.

Existem ainda diversos recursos que podem ser aplicados aos jogos para motivar o paciente. $14 \%$ dos trabalhos (16 artigos) fazem uso de outros elementos, tais como ferramentas de Realidade Virtual Imersiva, criação de contextos ou histórias envolventes para os 
jogos, questões relacionadas a feedback motivacional, humor e promoção de autonomia, dentre outros [Cuthbert et al. 2019, Bessa et al. 2020, Desai et al. 2016]. Lançando mão destas ferramentas, buscam prender o paciente à atividade, estabelecendo vínculos entre o paciente e o jogo.

\section{3. $\left(Q_{3}\right)$ Como se dá a interação entre o paciente e o jogo desenvolvido?}

Em relação à questão de pesquisa $Q_{3}$, o gráfico apresentado na Figura 2 (b) permite algumas observações. Estão sendo construídos jogos que utilizam diversos sistemas de interação. De todos os trabalhos selecionados, 26 (28\%) propõem uma interação baseada em gestos realizados pelo paciente, capturados por sensores instalados no ambiente, como o Kinect ${ }^{7} \mathrm{e}$ o Leap Motion Controller ${ }^{8}$ [Grammatikopoulou et al. 2019, Desai et al. 2016]. A facilidade de obtenção, dado que são equipamentos difundidos, e o baixo custo são elementos que têm sido relevantes para a utilização destes equipamentos em projetos de pesquisa.

Por outro lado, $46 \%$ dos trabalhos (43 artigos) trazem abordagens que utilizam sensores que precisam ser acoplados ao paciente, como goniometros ${ }^{9}$ e equipamentos de Eletroencefalograma (EEG) [Lai et al. 2018, Rahman et al. 2016]. É vantajosa a precisão dos dados capturados, porém estes equipamentos, além de possuírem alto custo e serem de difícil obtenção, precisam ser vestidos no paciente e configurados adequadamente, o que pode causar desconforto e perda da motivação em realizar a atividade.

Por fim, 24 trabalhos (26\%) baseiam-se em equipamentos que são utilizados pelo paciente, mas que não precisam ser fixados a ele [Hwang et al. 2017, López et al. 2017]. São controles utilizados em consoles de jogos, pedais adaptados, além de dispositivos desenvolvidos especificamente para os projetos. Dessa forma, pode-se reduzir algumas das dificuldades em relação à necessidade de que sejam acoplados sensores ao paciente.

\section{Considerações Finais}

Tendo como base a Revisão Sistemática apresentada, pode-se concluir que diversas iniciativas têm sido realizadas dentro da área de jogos aplicados à reabilitação de pacientes. São abordagens que utilizam sistemas de interação baseados em gestos ou ainda no uso de equipamentos, que podem ser acoplados ou não ao paciente. É essencial que, ao planejar o desenvolvimento da aplicação, leve-se em conta a praticidade e facilidade de uso e configuração dos dispositivos, além da precisão dos dados obtidos. É necessário um balanceamento neste sentido.

Em relação às abordagens que utilizam telerreabilitação, tem-se que poucas foram encontradas, ainda que existam diversos projetos que trabalham a reabilitação no contexto doméstico, sem a intervenção do fisioterapeuta. Considerando as grandes dificuldades de custo e acesso ao tratamento, bem como os cenários de pandemia e pós-pandemia da COVID-19 que impõem medidas de distanciamento social, é possível que, continuando-se a caminhada na direção em que está, a telerreabilitação se torne mais presente e com uma relevância cada vez maior.

Por outro lado, os trabalhos encontrados, ao concordarem acerca da importância do engajamento para o sucesso do tratamento, apontam para uma diversidade ainda maior de

\footnotetext{
${ }^{7}$ https://developer.microsoft.com/pt-br/windows/kinect/

${ }^{8} \mathrm{https} / / /$ www.ultraleap.com/product/leap-motion-controller/

${ }^{9} \mathrm{O}$ goniômetro ou sensor angular é uma espécie de sensor composto por dois componentes unidos por um cabo. Um componente é fixado imediatamente antes da articulação e o outro imediatamente depois. Conseguem medir a angulação de abertura da articulação.
} 
meios e recursos para que este engajamento seja instigado ou promovido dentro do jogo. A maioria massiva dos trabalhos aponta para o fato de que a própria construção do jogo já é fator que "prende" o paciente dentro da atividade, enquanto que outras iniciativas abordam recursos específicos que podem aumentar ainda mais esta sensação, como questões sobre a regulagem do nível de dificuldade das atividades ou ainda a definição de objetivos e metas, entre outros recursos. Esta visão reforça, por um lado, a importância das abordagens que envolvem jogos para a reabilitação e, por outro, que existem muitos elementos que podem ser utilizados e combinados para propiciar uma experiência melhor e mais engajadora para o paciente.

Alguns horizontes de pesquisa para trabalhos futuros podem ser delineados a partir desta revisão. Projetos que visem a construção de jogos em reabilitação podem considerar estas conclusões para a etapa de design da aplicação: a preocupação com a promoção de engajamento por meio da inserção de elementos no jogo e com as técnicas que visam a interação do paciente com ele. Dessa forma, os projetos desenvolvidos poderão contribuir de forma positiva com o cenário de reabilitação de pacientes baseada em jogos.

\section{Referências}

Afyouni, I., Einea, A., and Murad, A. (2019). Rehabot: Gamified virtual assistants towards adaptive telerehabilitation. In Adjunct Publication of the 27th Conference on User Modeling, Adaptation and Personalization, UMAP'19 Adjunct, page 21-26, New York, NY, USA. Association for Computing Machinery.

Agres, K. and Herremans, D. (2017). Music and motion-detection: A game prototype for rehabilitation and strengthening in the elderly. In 2017 International Conference on Orange Technologies (ICOT), pages 95-98.

Barioni, R. R., Chaves, T. M., Figueiredo, L., Teichrieb, V., Neto, E. V., and Da Gama, A. E. F. (2017). Arkanoidar: An augmented reality system to guide biomechanical movements at sagittal plane. In 2017 19th Symposium on Virtual and Augmented Reality (SVR), pages 207-214.

Bassett, S. (2003). The assessment of patient adherence to physiotherapy rehabilitation. New Zealand Journal of Physiotherapy, 31:60-66.

Bessa, D., Rodrigues, N. F., Oliveira, E., Kolbenschag, J., and Prahm, C. (2020). Designing a serious game for myoelectric prosthesis control. In 2020 IEEE 8th International Conference on Serious Games and Applications for Health (SeGAH), pages 1-5.

Bortone, I., Leonardis, D., Mastronicola, N., Crecchi, A., Bonfiglio, L., Procopio, C., Solazzi, M., and Frisoli, A. (2018). Wearable haptics and immersive virtual reality rehabilitation training in children with neuromotor impairments. IEEE Transactions on Neural Systems and Rehabilitation Engineering, 26(7):1469-1478.

Bower, K. J., Louie, J., Landesrocha, Y., Seedy, P., Gorelik, A., and Bernhardt, J. (2015). Clinical feasibility of interactive motion-controlled games for stroke rehabilitation. Journal of neuroengineering and rehabilitation, 12:63.

Brigham, T. J. (2015). An introduction to gamification: Adding game elements for engagement. Medical Reference Services Quarterly, 34(4):471-480. PMID: 26496401.

Burdea, G. C., Grampurohit, N., Kim, N., Polistico, K., Kadaru, A., Pollack, S., Oh-Park, M., Barrett, A., Kaplan, E., Masmela, J., and Nori, P. (2020). Feasibility of integrative games and novel therapeutic game controller for telerehabilitation of individuals chronic post-stroke living in the community. Topics in stroke rehabilitation, 27(5):321-336. 
Chatzitofis, A., Monaghan, D., Mitchell, E., Honohan, F., Zarpalas, D., O’Connor, N. E., and Daras, P. (2015). Hearthealth: A cardiovascular disease home-based rehabilitation system. Procedia Computer Science, 63:340-347.

Chebaa, B., Lioulemes, A., Abujelala, M., Ebert, D., Phan, S., Becker, E., and Makedon, F. (2016). Multimodal analysis of serious games for cognitive and physiological assessment. In Proceedings of the 9th ACM International Conference on PErvasive Technologies Related to Assistive Environments, PETRA '16, New York, NY, USA. Association for Computing Machinery.

Chen, W., Bang, M., Krivonos, D., Schimek, H., and Naval, A. (2020). An immersive virtual reality exergame for people with parkinson's disease. Lecture Notes in Computer Science (including subseries Lecture Notes in Artificial Intelligence and Lecture Notes in Bioinformatics), 12376 LNCS:138-145.

Chou, J. C., Hung, C., and Hung, Y. (2014). Design factors of mobile game for increasing gamer's flow experience. In 2014 IEEE International Conference on Management of Innovation and Technology, pages 137-139.

Corrêa, A. G. D., Kintschner, N. R., Campos, V. Z., and Blascovi-Assis, S. M. (2019). Gear vr and leap motion sensor applied in virtual rehabilitation for manual function training: An opportunity for home rehabilitation. In Proceedings of the 5th Workshop on ICTs for Improving Patients Rehabilitation Research Techniques, REHAB '19, page 148-151, New York, NY, USA. Association for Computing Machinery.

Csikszentmihalyi, M. (2020). Flow: A psicologia do alto desempenho e da felicidade. Objetiva.

Cuthbert, R., Turkay, S., and Brown, R. (2019). The effects of customisation on player experiences and motivation in a virtual reality game. In Proceedings of the 31st Australian Conference on Human-Computer-Interaction, OZCHI'19, page 221-232, New York, NY, USA. Association for Computing Machinery.

David, L., Bouyer, G., and Otmane, S. (2017). Towards an upper limb self-rehabilitation assistance system after stroke. In Proceedings of the Virtual Reality International Conference - Laval Virtual 2017, VRIC '17, New York, NY, USA. Association for Computing Machinery.

David, V., Forjan, M., Paštêka, R., Scherer, M., and Hofstätter, O. (2018). Development of a multi-purpose easy-to-use set of tools for home based rehabilitation: Use cases and applications developed during the rehabitation project. In Proceedings of the 8th International Conference on Software Development and Technologies for Enhancing Accessibility and Fighting Info-Exclusion, DSAI 2018, page 323-330, New York, NY, USA. Association for Computing Machinery.

Desai, K., Bahirat, K., Ramalingam, S., Prabhakaran, B., Annaswamy, T., and Makris, U. E. (2016). Augmented reality-based exergames for rehabilitation. In Proceedings of the 7th International Conference on Multimedia Systems, MMSys '16, New York, NY, USA. Association for Computing Machinery.

Dhawan, D., Barlow, M., and Lakshika, E. (2019). Prosthetic rehabilitation training in virtual reality. In 2019 IEEE 7th International Conference on Serious Games and Applications for Health (SeGAH), pages 1-8.

do Nascimento Piropo, T. G. and do Amaral, H. O. S. (2015). Telessaúde, contextos e implicações no cenário baiano. Saúde Debate, 39:279-287.

Eisapour, M., Cao, S., and Boger, J. (2020). Participatory design and evaluation of virtual reality games to promote engagement in physical activity for people living with dementia. Journal of rehabilitation and assistive technologies engineering, 7:2055668320913770. 
Ferguson, N. M., Laydon, D., Nedjati-Gilani, G., Imai, N., Ainslie, K., Baguelin, M., Bhatia, S., Boonyasiri, A., Cucunubá, Z., Cuomo-Dannenburg, G., Dighe, A., Dorigatti, I., Fu, H., Gaythorpe, K., Green, W., Hamlet, A., Hinsley, W., Okell, L. C., van Elsland, S., Thompson, H., Verity, R., Volz, E., Wang, H., Wang, Y., Walker, P. G., Walters, C., Winskill, P., Whittaker, C., Donnelly, C. A., Riley, S., and Ghani, A. C. (2020). Report 9: Impact of non-pharmaceutical interventions (npis) to reduce covid-19 mortality and healthcare demand. Imperial College COVID-19 Response Team, page 1-20.

Ferreira, B., Lourenço, J., and Menezes, P. (2019). A serious game for post-stroke motor rehabilitation. In 2019 5th Experiment International Conference (exp.at'19), pages 383387.

Ferreira, B. and Menezes, P. (2020). An adaptive virtual reality-based serious game for therapeutic rehabilitation. International journal of online and biomedical engineering, 16(4):63-71.

Funabashi, A. M. M., Aranha, R. V., Silva, T. D., Monteiro, C. B. M., Silva, W. S., and Nunes, F. L. S. (2017). Agar: A vr serious game to support the recovery of post-stroke patients. In 2017 19th Symposium on Virtual and Augmented Reality (SVR), pages 279288.

Grammatikopoulou, A., Dimitropoulos, K., Bostantjopoulou, S., Katsarou, Z., and Grammalidis, N. (2019). Motion analysis of parkinson diseased patients using a video game approach. In Proceedings of the 12th ACM International Conference on PErvasive Technologies Related to Assistive Environments, PETRA '19, page 523-527, New York, NY, USA. Association for Computing Machinery.

Huerga, R. S., Lade, J., and Mueller, F. (2016). Designing play to support hospitalized children. In Proceedings of the 2016 Annual Symposium on Computer-Human Interaction in Play, CHI PLAY '16, page 401-412, New York, NY, USA. Association for Computing Machinery.

Huizinga, J. (2020). Homo Ludens. Estudos. Editora Perspectiva S/A.

Hwang, S., Schneider, A. L. J., Clarke, D., Macintosh, A., Switzer, L., Fehlings, D., and Graham, T. N. (2017). How game balancing affects play: Player adaptation in an exergame for children with cerebral palsy. In Proceedings of the 2017 Conference on Designing Interactive Systems, DIS '17, page 699-710, New York, NY, USA. Association for Computing Machinery.

Jack, K., McLean, S. M., Moffett, J. K., and Gardiner, E. (2010). Barriers to treatment adherence in physiotherapy outpatient clinics: A systematic review. Manual Therapy, 15(3): $220-228$.

Kapp, K. M. (2012). The Gamification of Learning and Instruction: Game-based Methods and Strategies for Training and Education. Pfeiffer essential resources for training and HR professionals. Wiley.

Lai, Y., Sutjipto, S., Clout, M. D., Carmichael, M. G., and Paul, G. (2018). Gavre2: Towards data-driven upper-limb rehabilitation with adaptive-feedback gamification. In 2018 IEEE International Conference on Robotics and Biomimetics (ROBIO), pages 164-169.

López, S. A., Corno, F., and De Russis, L. (2017). Design and development of one-switch video games for children with severe motor disabilities. ACM Trans. Access. Comput., 10(4).

Madrigal, O. C., Rodríguez, Y. R., Díaz, A. A., and Kugurakova, V. (2019). Improving the communication model in game for health: Experiences in amblyopia treatment. In Proceedings of the 5th Workshop on ICTs for Improving Patients Rehabilitation Research 
Techniques, REHAB '19, page 5-9, New York, NY, USA. Association for Computing Machinery.

Nakagawa, E., Scannavino, K., Fabbri, S., and Ferrari, F. (2017). Revisão Sistemática da Literatura em Engenharia de Software: Teoria e Prática. Elsevier Brasil.

Oh, Y. and Yang, S. (2010). Defining exergames \& exergaming. In Proceedings of Meaningful Play 2010, pages 1-17. SBC.

Pedroli, E., Padula, P., Guala, A., Meardi, M., Riva, G., and Albani, G. (2017). A psychometric tool for a virtual reality rehabilitation approach for dyslexia. Computational and Mathematical Methods in Medicine, 2017.

Pereira, F., Bermúdez i Badia, S., Jorge, C., and da Silva Cameirão, M. (2019). Impact of game mode on engagement and social involvement in multi-user serious games with stroke patients. In 2019 International Conference on Virtual Rehabilitation (ICVR), pages $1-6$.

Rahman, M., Kankanhalli, A., Wadhwa, B., Hua, Y. C., Kei, C. K., Hoon, L. J., Jayakkumar, S., and Lin, C. C. (2016). Gear: A mobile game-assisted rehabilitation system. In 2016 IEEE International Conference on Healthcare Informatics (ICHI), pages 511-516.

Rodrigues, J., Menezes, P., and Restivo, M. T. (2019). Travelling in a virtual city: a physical exercise promoting game. In 2019 5th Experiment International Conference (exp.at'19), pages 256-257.

Sampaio, R. and Mancini, M. (2007). Estudos de revisão sistemática: Um guia para síntese criteriosa da evidência científica. Brazilian Journal of Physical Therapy, 11:83 - 89.

Santos, D. and Pinheiro, I. (2016). Telereabilitação no tratamento de disfunções neurológicas: Revisão narrativa. Revista Scientia, 1:96-106.

Silva, A. d. S. d., Valenciano, P. J., and Fujisawa, D. S. (2017). Atividade Lúdica na Fisioterapia em Pediatria: Revisão de Literatura. Revista Brasileira de Educação Especial, 23:623- 636 .

Souza, C. H. R., Battisti, D., Berretta, L., and de Carvalho, S. T. (2020). Exergame com cicloergômetro para a reabilitação de pacientes em tempos de covid-19. In Anais Principais do XX Simpósio Brasileiro de Computação Aplicada à Saúde, Porto Alegre, RS, Brasil. SBC.

Subtil, M. M. L., Goes, D. C., Gomes, T. C., and Souza, M. L. d. (2011). O relacionamento interpessoal e a adesão na fisioterapia. Fisioterapia em Movimento, 24:745 - 753.

Tamayo-Serrano, P., Garbaya, S., Bouakaz, S., and Blazevic, P. (2020). A game-based rehabilitation therapy for post-stroke patients: An approach for improving patient motivation and engagement. IEEE Systems, Man, and Cybernetics Magazine, 6(4):54-62.

Tolomei, B. V. (2017). A gamificação como estratégia de engajamento e motivação na educação. EaD em Foco, 7(2).

Uzor, S. and Baillie, L. (2019). Recov-r: Evaluation of a home-based tailored exergame system to reduce fall risk in seniors. ACM Trans. Comput.-Hum. Interact., 26(4).

Vasconcelos, A., Nunes, F., Carvalho, A., and Correia, C. (2018). Mobile, exercise-agnostic, sensor-based serious games for physical rehabilitation at home. In Proceedings of the Twelfth International Conference on Tangible, Embedded, and Embodied Interaction, TEI '18, page 271-278, New York, NY, USA. Association for Computing Machinery. 